\title{
Correspondence
}

\section{Nonbinary LDPC-Coded Sphere-Packed Transmit Diversity}

\author{
Osamah Alamri, Soon X. Ng, Feng Guo, Member, IEEE, \\ Salam Zummo, and Lajos Hanzo, Fellow, IEEE
}

\begin{abstract}
A recently proposed space-time block-coding (STBC) signal construction method that combines orthogonal design with sphere packing (SP) (referred to here as STBC-SP) has shown useful performance improvements over Alamouti's conventional orthogonal design. In this paper, we propose a purely symbol-based, low-density parity-check (LDPC)-coded scheme, demonstrating that the performance of STBC-SP systems can further be improved by concatenating SP-aided modulation with nonbinary LDPC and by performing symbol-based turbo detection between the nonbinary LDPC decoder and a rate-1 nonbinary inner precoder. We also investigate the convergence behavior of this symbol-based concatenated scheme with the aid of novel nonbinary extrinsic information transfer (EXIT) charts. Finally, we demonstrate that in the investigated scenarios, it requires 1-2 dB lower power in comparison with the equivalent effectivethroughput 0.5-, 0.75-, and 1-bit/symbol systems employing bit-based turbo detection.
\end{abstract}

Index Terms-Nonbinary low-density parity check (LDPC), space-time codes, sphere packing (SP), symbol-based extrinsic information transfer (EXIT) chart, turbo detection.

\section{INTRODUCTION}

The adverse effects of channel fading may significantly be reduced by employing space-time (ST) coding that invokes multiple antennas [1]. Alamouti [2] discovered an appealingly simple transmit diversity scheme employing two transmit antennas. This low-complexity design inspired Tarokh et al. [3] to generalize Alamouti's transmit diversity scheme using the principle of orthogonal design to an arbitrary number of transmit antennas. The concept of combining orthogonal transmit diversity designs with the principle of sphere packing (SP) was introduced by Su et al. in [4], where it was demonstrated that the proposed SP-aided ST block-coded (STBC) system (referred to here as STBCSP) was capable of outperforming the conventional orthogonal designbased STBC schemes in [2] and [3].

Surprisingly, the family of low-density parity-check (LDPC) codes originally devised by Gallager as early as 1963 [5] remained more or less unexploited until after the discovery of turbo codes in 1993 [6]. Since then, however, LDPC codes have experienced a renaissance and attracted substantial research interest. In 1998, Davey and MacKay proposed a nonbinary version of LDPC codes [7], which was potentially capable of outperforming binary LDPC codes.

Manuscript received July 19, 2007; revised November 1, 2007 and December 4, 2007. This work was supported in part by the Engineering and Physical Sciences Research Council, U.K., by the European Union under the Optimix project, and by the Ministry of Higher Education of Saudi Arabia. The review of this paper was coordinated by Dr. A. Ghrayeb.

O. Alamri, S. X. Ng, F. Guo, and L. Hanzo are with the University of Southampton, Southampton, SO17 1BJ Hampshire, U.K. (e-mail: ora02r@ ecs.soton.ac.uk; sxn@ecs.soton.ac.uk; fg01r@ecs.soton.ac.uk; lh@ecs.soton. ac.uk).

S. Zummo is with King Fahd University of Petroleum and Minerals, Dhahran, Eastern Province 31261, Saudi Arabia (e-mail: zummo@kfupm. edu.sa).

Digital Object Identifier 10.1109/TVT.2008.917217
It was also shown in [8] that a recursive rate-1 inner code is beneficial to maximize the interleaver gain and, hence, to avoid a bit error ratio (BER) floor when employing iterative decoding. In [9], it was shown that a reduced transmit power may be required when symbol-based rather than bit-based iterative decoding is employed.

Motivated by the performance improvements reported in [4] and [7]-[9], we propose a novel purely symbol-based iterative scheme. We will demonstrate that the proposed nonbinary turbo-detectionaided STBC-SP scheme is capable of providing further performance improvements over both the STBC-SP scheme in [4] as well as over a bit-based LDPC-coded, turbo-detected STBC-SP scheme. The novel nonbinary extrinsic information transfer (EXIT) characteristic charts in [10] and [11] are employed to design our nonbinary scheme. The rationale of the proposed architecture is explicit.

1) SP modulation maximizes the coding advantage of the transmission scheme by jointly-rather than separately-designing and detecting the SP symbols hosting the two time-slots' STBC symbols. ${ }^{1}$

2) The inner rate- 1 encoder and its low-complexity recursive decoder beneficially distributes the extrinsic information without reducing the effective throughput, maximizes the interleaver gain at a given length, and, hence, avoids having a BER floor.

3) Symbol-based iterative SP detection and decoding outperforms its bit-based counterpart.

This paper is organized as follows. A brief description of our system is presented in Section II. An overview of orthogonal STBC design using SP modulation is provided in Section III. Symbol-based iterative decoding is described in Section IV. Section V provides our EXIT chart analysis, whereas our simulation results are discussed in Section VI. Finally, we conclude in Section VII.

\section{SySTEM OVERVIEW}

In this section, we will describe the proposed turbo-detected symbol-based scheme as well as its bit-based counterpart. The schematic of the nonbinary arrangement is shown in Fig. 1. The source bits are encoded by a rate $R=1 / 2$ nonbinary LDPC encoder [7] to generate the LDPC-encoded symbols $\mathbf{v}=\left\{v_{0}, v_{1}, \ldots, v_{K_{\mathrm{ldpc}}-1}\right\}$, $v_{k} \in G F(q)$, where $K_{\mathrm{ldpc}}$ is the LDPC output block length, and $q$ is the size of the LDPC decoding field. In this paper, we consider randomly constructed nonbinary LDPC codes [7] having an average column weight of $\omega_{l}$ and employing Richardson's fast-Fouriertransform-based decoding algorithm [12]. The LDPC-encoded symbols are then precoded by a nonbinary rate- 1 encoder before each of them is mapped to the corresponding SP-modulated symbol $\mathbf{s}^{l} \in S$, $0 \leq l \leq L-1$. There is a natural one-to-one mapping between $l$ and the elements of the nonbinary LDPC code defined over $G F(q)$, where we have $L=q$, allowing us to create a purely symbol-based system. Again, the rate-1 precoder shown in Fig. 1 is also a nonbinary encoder, which is defined by the binary generator polynomial $G=\left(g / g_{r}\right)=$ $(10 / 11)$, where $g$ denotes the feedforward output, and $g_{r}$ is the feedback to the input using modulo $q$ addition. Observe that channel interleaving is not required between the nonbinary LDPC encoder and the rate- 1 encoder since the LDPC parity check matrix is randomly

\footnotetext{
${ }^{1}$ By contrast, Alamouti detected two seemingly independent QPSK ST symbols, although their amalgam constitutes a combined symbol.
} 

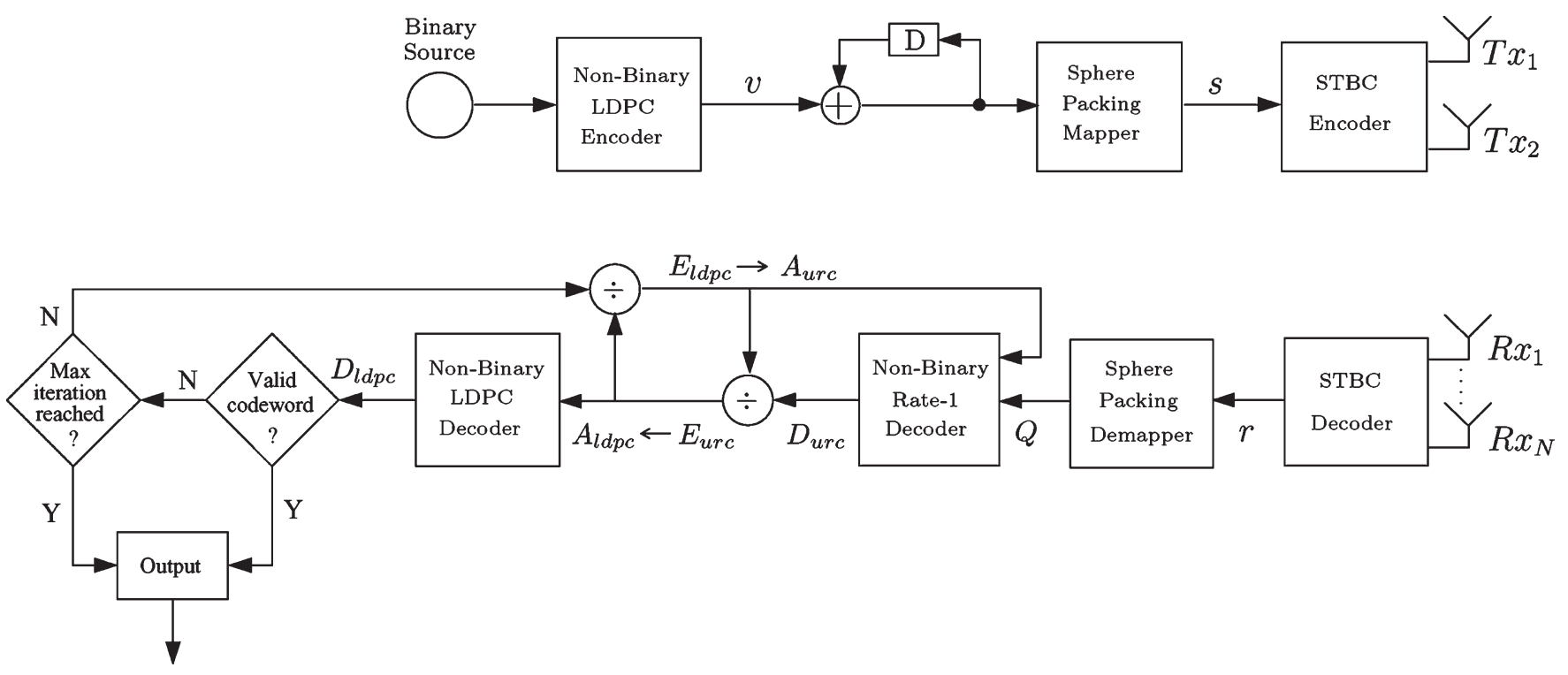

Fig. 1. Symbol-based turbo detection STBC-SP system.

constructed, where each of the parity check equations is checking several random $G F(q)$ symbol positions in a codeword, which has a similar effect to that of the channel interleaver. The STBC encoder then maps each SP-modulated symbol $\mathbf{s}^{l} \in S$ to an ST signal $\mathbf{C}_{l}$, $0 \leq l \leq L-1$. Subsequently, each STBC-SP symbol is transmitted over $T=2$ consecutive time slots using two transmit antennas. The details and motivation of SP-aided STBC are discussed in Section III.

In this treatise, we considered an uncorrelated narrowband Rayleigh fading channel based on the Jakes fading model. The complex fading envelope is assumed to be constant across the transmission period of an ST-coded symbol spanning $T=2$ time slots. The complex additive white Gaussian noise (AWGN) of $n=n_{I}+j n_{Q}$ is also added to the received signal, where $n_{I}$ and $n_{Q}$ are two independent zero-mean Gaussian random variables having a variance of $\sigma_{n}^{2}=\sigma_{n_{I}}^{2}=\sigma_{n_{Q}}^{2}=$ $N_{0} / 2$ per dimension, where $N_{0} / 2$ represents the double-sided noise power spectral density.

As shown in Fig. 1, the received complex-valued symbols are first decoded by the STBC decoder to produce a received SP symbol $\mathbf{r}$, which is fed into the SP demapper, where the soft metric $\mathbf{Q}(k)$ is calculated. More explicitly, the notation $\mathbf{Q}(k)$ represents the soft metric passed from the SP demapper to the nonbinary rate- 1 decoder based on the probability of the $k$ th symbol of the encoded codeword by the rate-1 encoder, as will be shown in (7). As shown in Fig. 1, the rate-1 decoder processes these soft metrics in conjunction with the a priori information $\mathbf{A}_{\mathrm{urc}}$ to generate the a posteriori probability (APP) $\mathbf{D}_{\text {urc }}$, where the subscript "urc" refers to the unity rate code. After removing the a priori information $\mathbf{A}_{\text {urc }}$ from the APP denoted by $\mathbf{D}_{\text {urc }}, \mathbf{A}_{\mathrm{ldpc}}$ is passed as a priori information to the LDPC decoder, which carries out a specified number of LDPC iterations and produces the decoded APP $\mathbf{D}_{\text {ldpc }}$. Based on the APP, a tentative hard decision will be made, and the resultant codeword will be checked by the LDPC code's parity check matrix. If the resultant vector is an all-zero sequence, then a legitimate codeword has been found, and the harddecision-based sequence will be outputted. Otherwise, if the maximum affordable number of iterations has not been reached, the a priori information $\mathbf{A}_{\text {ldpc }}$ is removed from the APP denoted by $\mathbf{D}_{\text {ldpc }}$.

The structure of the bit-based scheme is identical to its symbolbased counterpart shown in Fig. 1, except that a binary-rather than nonbinary-LDPC code is invoked to investigate the employment of bit interleaving and bit-based iterative decoding. We consider a randomly constructed binary LDPC code [5] having an average column weight of $\omega_{l}$. A bit-to-symbol probability conversion is required when passing extrinsic information from the binary LDPC decoder to the rate-1 decoder. On the other hand, symbol-to-bit probability conversion is required when passing extrinsic information from the rate- 1 decoder to the binary LDPC decoder.

\section{ORthogonal Design With SP Modulation}

This section briefly reviews the STBC-SP scheme proposed in [4]. In this contribution, ST systems employing two transmit antennas are considered, where the ST signal is given by [2]

$$
\mathbf{G}_{2}\left(x_{1}, x_{2}\right)=\left[\begin{array}{cc}
x_{1} & x_{2} \\
-x_{2}^{*} & x_{1}^{*}
\end{array}\right]
$$

and the rows and columns represent the temporal and spatial dimensions, which correspond to two consecutive time slots and two transmit antennas, respectively. According to Alamouti's design [2], for example, $x_{1}$ and $x_{2}$ represent conventional BPSK-modulated symbols transmitted in the first and second time slots, and no effort is made to jointly design a signal constellation for the various combinations of $x_{1}$ and $x_{2}$. It was shown, however, in [4] that the diversity product quantifying the coding advantage ${ }^{2}$ of an orthogonal transmit diversity scheme is determined by the minimum Euclidean distance (MED) of the various vectors $\left(x_{1}, x_{2}\right)$. Therefore, to maximize the achievable coding advantage, it was proposed in [4] to use SP schemes that have the best-known MED in the 4-D real-valued Euclidean space $\mathbb{R}^{4}[13]$. For the sake of generalizing our treatment, let us assume that there are $L$ legitimate ST signals $\mathbf{G}_{2}\left(x_{l, 1}, x_{l, 2}\right), l=0,1, \ldots, L-1$, where $L$ represents the number of SP-modulated symbols. The transmitter then has to choose the modulated signal from these $L$ legitimate symbols, which have to be transmitted over the two antennas in two consecutive time slots, where the throughput of the system is given by $\left(\log _{2} L\right) / 2$ bits per channel use. In contrast to Alamouti's independent design of the two time slots' signals, our aim is to jointly design $x_{l, 1}$ and $x_{l, 2}$ so that

\footnotetext{
${ }^{2}$ The diversity product or coding advantage was defined as the estimated gain over an uncoded system having the same diversity order as the coded system [4].
} 
they have the best MED from all other $(L-1)$ legitimate transmitted ST signals, since this minimizes the system's error probability. Let $\left(a_{l, 1}, a_{l, 2}, a_{l, 3}, a_{l, 4}\right), l=0,1, \ldots, L-1$ be phasor points from the 4-D real-valued Euclidean space $\mathbb{R}^{4}$, where each of the four elements $a_{l, 1}, a_{l, 2}, a_{l, 3}$, and $a_{l, 4}$ gives one coordinate of the two time slots' complex-valued phasor points. Hence, $x_{l, 1}$ and $x_{l, 2}$ may be written as

$$
\begin{aligned}
\left\{x_{l, 1}, x_{l, 2}\right\} & =T_{s p}\left(a_{l, 1}, a_{l, 2}, a_{l, 3}, a_{l, 4}\right) \\
& =\left\{a_{l, 1}+j a_{l, 2}, a_{l, 3}+j a_{l, 4}\right\} .
\end{aligned}
$$

In the 4-D real-valued Euclidean space $\mathbb{R}^{4}$, the lattice $D_{4}$ is defined as an SP constellation having the best MED from all the other $(L-1)$ legitimate constellation points in $\mathbb{R}^{4}$ [13]. More specifically, $D_{4}$ may be defined as a lattice that consists of all legitimate SP constellation points having integer coordinates $\left[a_{1} a_{2} a_{3} a_{4}\right]$ uniquely and unambiguously describing the legitimate combinations of the two time slots' modulated symbols in Alamouti's scheme but subjected to the SP constraint of $a_{1}+a_{2}+a_{3}+a_{4}=k$, where $k$ is an even integer. Assuming that $S=\left\{\mathbf{s}^{l}=\left[a_{l, 1} a_{l, 2} a_{l, 3} a_{l, 4}\right] \in \mathbb{R}^{4}: 0 \leq l \leq\right.$ $L-1\}$ constitutes a set of $L$ legitimate constellation points from the lattice $D_{4}$ having a total energy of $E \triangleq \sum_{l=0}^{L-1}\left(\left|a_{l, 1}\right|^{2}+\left|a_{l, 2}\right|^{2}+\right.$ $\left.\left|a_{l, 3}\right|^{2}+\left|a_{l, 4}\right|^{2}\right)$, and upon introducing the notation

$$
\mathbf{C}_{l}=\sqrt{\frac{2 L}{E}} \mathbf{G}_{2}\left(x_{l, 1}, x_{l, 2}\right), \quad l=0,1, \ldots, L-1
$$

we have a set of ST signals $\left\{\mathbf{C}_{l}: 0 \leq l \leq L-1\right\}$ whose diversity product is determined by the MED of the set of $L$ legitimate SP constellation points in $S$.

\section{Symbol-BASEd ITERATIVE DECODING}

For the sake of simplicity, a system having a single receive antenna is considered, although its extension to systems having more than one receive antenna is straightforward. Assuming perfect channel estimation, the complex-valued channel output symbols received during two consecutive time slots are first diversity combined to extract the estimates $\tilde{x}_{1}$ and $\tilde{x}_{2}$ of the most likely transmitted symbols $x_{l, 1}$ and $x_{l, 2}$ [1, pp. 400-401], [2], resulting in $\tilde{x}_{i}=\left(\left|h_{1}\right|^{2}+\left|h_{2}\right|^{2}\right) \cdot x_{l, i}+$ $\dot{n}_{i}$ for $i=1,2 . h_{1}$ and $h_{2}$ represent the complex-valued channel coefficients corresponding to the first and second transmit antennas, respectively, and $\dot{n}_{1}$ and $\dot{n}_{2}$ are zero-mean complex Gaussian random variables with a variance of $\sigma_{\tilde{n}}^{2}=\left(\left|h_{1}\right|^{2}+\left|h_{2}\right|^{2}\right) \cdot \sigma_{n}^{2}$. A received SP symbol $\mathbf{r}$ is then constructed from the estimates $\tilde{x}_{1}$ and $\tilde{x}_{2}$, where $\mathbf{r}=$

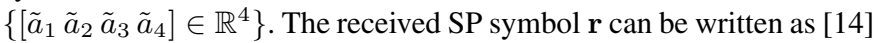

$$
\mathbf{r}=h \cdot \sqrt{\frac{2 L}{E}} \cdot \mathbf{s}^{l}+\mathbf{w}
$$

where $h=\left(\left|h_{1}\right|^{2}+\left|h_{2}\right|^{2}\right), \mathbf{s}^{l} \in S, 0 \leq l \leq L-1$, and $\mathbf{w}$ is a 4-D real-valued Gaussian random variable having a covariance matrix of $\sigma_{w}^{2} \cdot \mathbf{I}_{N_{D}}=\sigma_{\grave{n}}^{2} \cdot \mathbf{I}_{N_{D}}=h \cdot \sigma_{n}^{2} \cdot \mathbf{I}_{N_{D}}$, where $N_{D}=4$, since the symbol constellation $S$ is 4-D. According to (4), the conditional probability density function (pdf) $p\left(\mathbf{r} \mid \mathbf{s}^{l}\right)$ is given by

$$
\begin{aligned}
p\left(\mathbf{r} \mid \mathbf{s}^{l}\right) & =\frac{1}{\left(2 \pi \sigma_{w}^{2}\right)^{\frac{N_{D}}{2}}} e^{-\frac{1}{2 \sigma_{w}^{2}}\left(\mathbf{r}-\alpha \cdot \mathbf{s}^{l}\right)\left(\mathbf{r}-\alpha \cdot \mathbf{s}^{l}\right)^{T}} \\
& =\frac{1}{\left(2 \pi \sigma_{w}^{2}\right)^{\frac{N_{D}}{2}}} e^{-\frac{1}{2 \sigma_{w}^{2}}\left(\sum_{i=1}^{4}\left(\tilde{a}_{i}-\alpha \cdot a_{l, i}\right)^{2}\right)}
\end{aligned}
$$

where we have $\alpha=h \cdot \sqrt{2 L / E}$, and $(\cdot)^{T}$ represents the transpose of a vector.
Similarly, the conditional pdf $p\left(\mathbf{s}^{l} \mid \mathbf{r}\right)$ is given by

$$
p\left(\mathbf{s}^{l} \mid \mathbf{r}\right)=\frac{p\left(\mathbf{r} \mid \mathbf{s}^{l}\right) \cdot p\left(\mathbf{s}^{l}\right)}{p(\mathbf{r})} .
$$

Since the LDPC codeword consists of $K_{\mathrm{ldpc}} G F(q)$ symbols, the SP demapper in Fig. 1 will process $K_{\text {ldpc }}$ received SP symbols $\left(\mathbf{r}_{0}, \mathbf{r}_{1}, \ldots, \mathbf{r}_{K_{\mathrm{ldpc}}-1}\right)$ at a time to produce the following $\left(K_{\mathrm{ldpc}} \times L\right)$ soft-metric matrix using (6):

$$
\mathbf{Q}=\left[\begin{array}{llll}
\mathbf{Q}(0) & \mathbf{Q}(1) & \cdots & \mathbf{Q}\left(K_{\mathrm{ldpc}}-1\right)
\end{array}\right]^{T}
$$

where $\mathbf{Q}(k)=\left[p\left(\mathbf{s}_{k}=\mathbf{s}^{0} \mid \mathbf{r}_{k}\right) p\left(\mathbf{s}_{k}=\mathbf{s}^{1} \mid \mathbf{r}_{k}\right) \cdots p\left(\mathbf{s}_{k}=\mathbf{s}^{L-1} \mid \mathbf{r}_{k}\right)\right]$ for $k=0,1, \ldots, K_{\mathrm{ldpc}}-1$. All the probabilities corresponding to a specific row in $\mathbf{Q}$, which correspond to a specific received symbol, should be normalized so that they sum up to unity.

The nonbinary rate- 1 decoder in Fig. 1 then processes the softmetric matrix $\mathbf{Q}$ of (7) in conjunction with the a priori information $\mathbf{A}_{\text {urc }}$ to produce a decoded APP matrix $\mathbf{D}_{\text {urc }}$ of size $\left(K_{\text {ldpc }} \times L\right)$ using a standard implementation of the forward-backward recursionbased APP algorithm [15]. During the first iteration, $p\left(\mathbf{s}^{l}\right), 0 \leq l \leq$ $L-1$, which is shown in (6), has to be set to $1 / q$ since no a priori information is available from the LDPC decoder. The a priori knowledge fed into the rate- 1 decoder in Fig. 1 is removed from the decoded APP matrix $\mathbf{D}_{\text {urc }}$ using symbol-based element-wise division [10] for the sake of generating the extrinsic probability matrix $\mathbf{E}_{\mathrm{urc}}$, which is then fed into the LDPC decoder as the a priori knowledge $\mathbf{A}_{\mathrm{ldpc}}$, as alluded to before. More specifically, the following $\left(K_{\mathrm{ldpc}} \times L\right)$ a priori information matrix is constructed:

$$
\mathbf{A}_{\mathrm{ldpc}}=\left[\begin{array}{llll}
\mathbf{A}_{\mathrm{ldpc}}(0) & \mathbf{A}_{\mathrm{ldpc}}(1) & \cdots & \mathbf{A}_{\mathrm{ldpc}}\left(K_{\mathrm{ldpc}}-1\right)
\end{array}\right]^{T}
$$

where $\mathbf{A}_{\text {ldpc }}(k)=\left[p\left(\mathbf{s}_{k}=\mathbf{s}^{0}\right) p\left(\mathbf{s}_{k}=\mathbf{s}^{1}\right) \cdots p\left(\mathbf{s}_{k}=\mathbf{s}^{L-1}\right)\right]$, and we have $p\left(\mathbf{s}_{k}=\mathbf{s}^{l}\right)=\left(d_{\mathrm{urc}}\right)_{k}^{l} /\left(a_{\mathrm{urc}}\right)_{k}^{l}, 0 \leq l \leq L-1$ and $0 \leq k \leq$ $K_{\mathrm{ldpc}}-1$, whereas $\left(d_{\mathrm{urc}}\right)_{k}^{l}$ and $\left(a_{\mathrm{urc}}\right)_{k}^{l}$ refer to the elements at the crossover point of the $k$ th row and $l$ th column of the matrices $\mathbf{D}_{\text {urc }}$ and $\mathbf{A}_{\text {urc }}$, respectively. Again, the probabilities corresponding to a specific row of the matrix $\mathbf{A}_{\text {ldpc }}$ should be normalized so that the values add up to unity. The LDPC decoder exploits the a priori information $\mathbf{A}_{\text {ldpc }}$ for the sake of producing a decoded soft metric $\mathbf{D}_{\text {ldpc }}$. Again, the a priori information $\mathbf{A}_{\text {ldpc }}$ is removed from the decoded APP matrix $\mathbf{D}_{\text {ldpc }}$ by symbol-based element-wise division for the sake of generating $\mathbf{E}_{\mathrm{ldpc}}$, which is passed to the rate-1 decoder in Fig. 1 as the a priori knowledge $\mathbf{A}_{\text {urc }}$ for further iterations until a legitimate codeword is found or the affordable maximum number of iterations has been exhausted.

\section{NONBINARY EXIT CHART ANALYSIS}

\section{A. Theoretical Background}

The main objective of employing EXIT charts [16] is to predict the convergence behavior of the iterative decoder by examining the evolution of the input/output mutual information exchange between the inner and outer decoders in consecutive iterations. Denoting the mutual information between two random variables $X$ and $Y$ as $I(X ; Y)$, the average a priori information $I_{A_{\mathrm{urc}}}$ at the input of the inner nonbinary rate-1 decoder and the average extrinsic information $I_{E_{\mathrm{urc}}}$ at the output of the inner nonbinary rate-1 decoder can be defined as [17]

$$
\begin{aligned}
& I_{A_{\mathrm{urc}}}:=\frac{1}{M} \sum_{i=0}^{M-1} I\left(V_{i} ; \mathbf{A}_{\mathrm{urc}}(i)\right) \\
& I_{E_{\mathrm{urc}}}:=\frac{1}{M} \sum_{i=0}^{M-1} I\left(V_{i} ; \mathbf{E}_{\mathrm{urc}}(i)\right)
\end{aligned}
$$


where $V_{i}$ is an $L$-ary random variable representing the $i$ th integer symbol $v_{i}$ at the input of the rate-1 encoder in Fig. 1, and $M$ is the total number of legitimate symbols $v_{i}$. Note that $\mathbf{A}_{\text {urc }}(i)$ and $\mathbf{E}_{\text {urc }}(i)$ are vectors of random variables corresponding to the $i$ th row of the matrices $\mathbf{A}_{\text {urc }}$ and $\mathbf{E}_{\text {urc }}$, respectively. The transfer characteristic $T_{\text {urc }}$ of the inner rate- 1 decoder is a function of $I_{A_{\text {urc }}}$ and $E_{b} / N_{0}$ expressed as $I_{E_{\mathrm{urc}}}=T_{\mathrm{urc}}\left(I_{A_{\mathrm{urc}}}, E_{b} / N_{0}\right)$. Similarly, the average a priori information $I_{A_{\mathrm{ldpc}}}$ at the input of the outer nonbinary LDPC decoder and the average extrinsic information $I_{E_{1 \mathrm{dpc}}}$ at the output of the outer nonbinary LDPC decoder can be defined as

$$
\begin{aligned}
I_{A_{\mathrm{ldpc}}} & :=\frac{1}{M} \sum_{i=0}^{M-1} I\left(V_{i} ; \mathbf{A}_{\mathrm{ldpc}}(i)\right) \\
I_{E_{\mathrm{ldpc}}} & :=\frac{1}{M} \sum_{i=0}^{M-1} I\left(V_{i} ; \mathbf{E}_{\mathrm{ldpc}}(i)\right)
\end{aligned}
$$

where the transfer characteristic $T_{\mathrm{ldpc}}$ of the outer nonbinary LDPC decoder is given by $I_{E_{\mathrm{ldpc}}}=T_{\mathrm{ldpc}}\left(I_{A_{\mathrm{ldpc}}}\right)$, which does not depend on the $E_{b} / N_{0}$ values.

It was shown in [11] that by averaging over a sufficiently large number of length $K_{\mathrm{ldpc}}$ blocks, the mutual information $I_{E}$ can be estimated as

$$
I_{E}=-H\left(V_{1}\right)+E\left\{\frac{1}{K_{\mathrm{ldpc}}} \sum_{i=0}^{K_{\mathrm{ldpc}}-1} \sum_{l=0}^{L-1} e_{k}^{l}\right\}
$$

where $e_{k}^{l}$ refers to the element at the crossover point of the $k$ th row and $l$ th column of the matrices $\mathbf{E}_{\mathrm{urc}}$ or $\mathbf{E}_{\mathrm{ldpc}}$, and the entropy $H\left(V_{1}\right)$ can readily be determined from the a priori $L$-ary symbol distributions $p\left(v_{i}\right)$. For example, if we have $p\left(v_{i}=l\right)=1 / L$ for $l=0,1, \ldots, L-1$ (i.e., equiprobable $L$-ary symbols), then we arrive at $H\left(V_{1}\right)=-\log _{2}(L)$.

\section{B. EXIT Chart Results}

Fig. 2 shows the EXIT chart of the symbol-based LDPC-coded STBC-SP scheme (Fig. 1) in combination with $L=4$ and the $1 / 2$ rate outer LDPC code [7] defined over $G F(4)$ when operating at a signal-to-noise ratio (SNR) of $0.5 \mathrm{~dB}$ and using $I_{\text {int }}=3$ internal LDPC iterations as well as the system parameters outlined in Table I. This is referred to as Scheme 1. Observe in Fig. 2 that the maximum mutual information is $\log _{2}(L)=2$, which is also evident from (11). Fig. 2 also shows the EXIT chart of an identical-throughput $1 / 2$ bitper-symbol bit-based LDPC-coded STBC-SP scheme in combination with the 1/2-rate outer LDPC code defined over $G F(2)$. Binary EXIT charts [16] are employed to study the convergence behavior of bitbased schemes. Ideally, for the exchange of extrinsic information between the rate-1 decoder and the outer LDPC decoder in Fig. 1 to converge at a specific SNR value, the EXIT curve of the rate-1 decoder recorded at the SNR value of interest and that of the outer LDPC decoder should only intersect at the point of $\left(I_{A}, I_{E}\right)=(2.0,2.0)$. If this condition is satisfied, then a so-called open convergence tunnel [16] appears in the EXIT chart. The narrower the tunnel, the closer the system's performance to the channel capacity, and hence, in the spirit of Shannon's information theory, more iterations are required to reach the $(2.0,2.0)$ point.

Observe in Fig. 2 that the symbol-based Scheme 1 in Table I exhibits an open convergence tunnel at $\mathrm{SNR}=0.5 \mathrm{~dB}$, whereas the equivalent bit-based scheme requires higher SNR values before an open convergence tunnel can be formed. This implies that according to the predictions of the EXIT chart shown in Fig. 2, the symbol-based

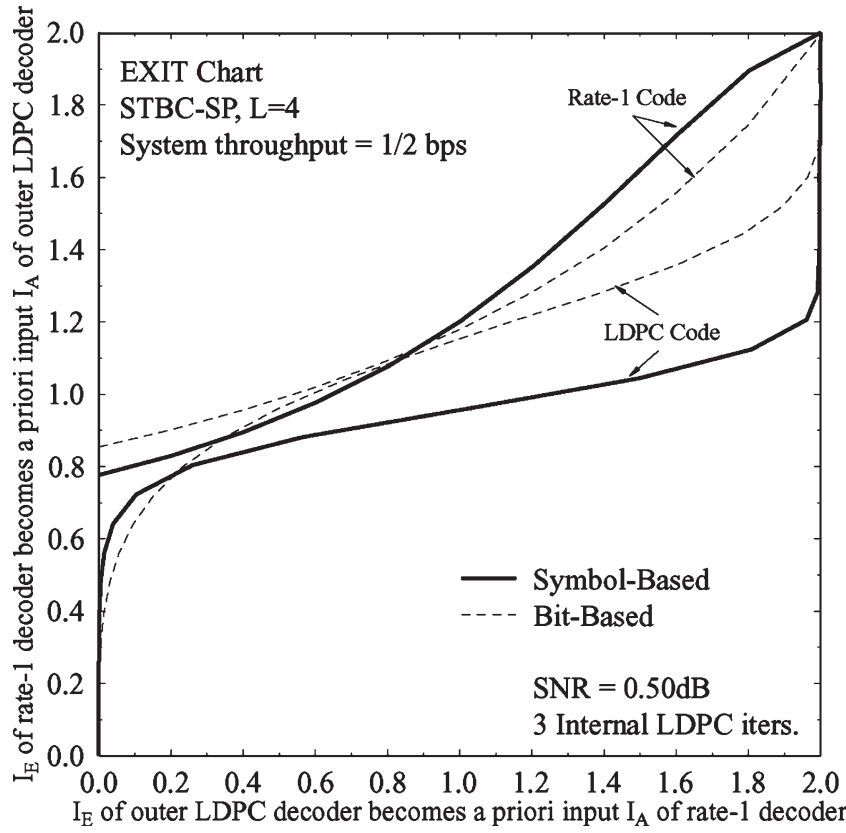

Fig. 2. EXIT chart of symbol-based and bit-based LDPC-coded STBC-SP schemes in combination with the $1 / 2$-rate outer LDPC code defined over $G F(4)$ and $G F(2)$, respectively, using three internal LDPC iterations and the system parameters outlined in Table I

TABLE I

SYSTEM PARAMETERS

\begin{tabular}{|l||c|c|c|}
\hline \multicolumn{1}{|c||}{} & Scheme 1 & Scheme 2 & Scheme 3 \\
\hline SP constellation size & $L=4$ & $L=8$ & $L=16$ \\
\hline No. of transmitters & \multicolumn{3}{|c|}{$N_{T X}=2$} \\
\hline No. of receivers & \multicolumn{3}{c|}{$N_{R X}=1$} \\
\hline Channel & \multicolumn{3}{|c|}{$\omega_{l}=2.5$} \\
\hline Average LDPC column weight & \multicolumn{3}{|c|}{$R=\frac{1}{2}$} \\
\hline LDPC coding rate & $G F(4)$ & $G F(8)$ & $G F(16)$ \\
\hline Non-Binary LDPC decoding field & $G F(4)$ & $G F(8)$ & $G F(16)$ \\
\hline Rate-1 decoding field & $1 / 2$ & $3 / 4$ & 1 \\
\hline System throughput (BPS) & $K_{l d p c}=6000$ to 12000 bits \\
\hline LDPC Coded Blocklength
\end{tabular}

Scheme 1 in Table I is expected to have a lower convergence threshold than its bit-based counterpart, and hence, the former will exhibit a BER turbo cliff at a lower SNR value.

Fig. 3 shows the EXIT chart of the symbol-based Scheme 3 in Table I when using $I_{\text {int }}=3$ internal LDPC iterations and operating at SNR of $4.50 \mathrm{~dB}$. Fig. 3 also shows the EXIT chart of the equivalentthroughput bit-based scheme. Observe that although both symbolbased and bit-based schemes require similar SNR values to exhibit an open convergence tunnel, the symbol-based scheme exhibits a wider tunnel. Hence, a lower number of iterations is needed to reach the convergence point of $\left(I_{A}, I_{E}\right)=(4.0,4.0)$. These EXIT tunnelbased convergence predictions are usually verified by the actual EXIT trajectory of iterative decoding as well as by the BER curves, as will be discussed in Section VI.

\section{RESUlTS AND Discussion}

Without loss of generality, we considered an SP modulation scheme associated with $L=4,8$, and 16 using two transmit and a single receiver antenna to demonstrate the performance improvements achieved by the proposed system. All simulation parameters are listed in Table I. There are more than $L$ legitimate SP symbols in the lattice $D_{4}$, 


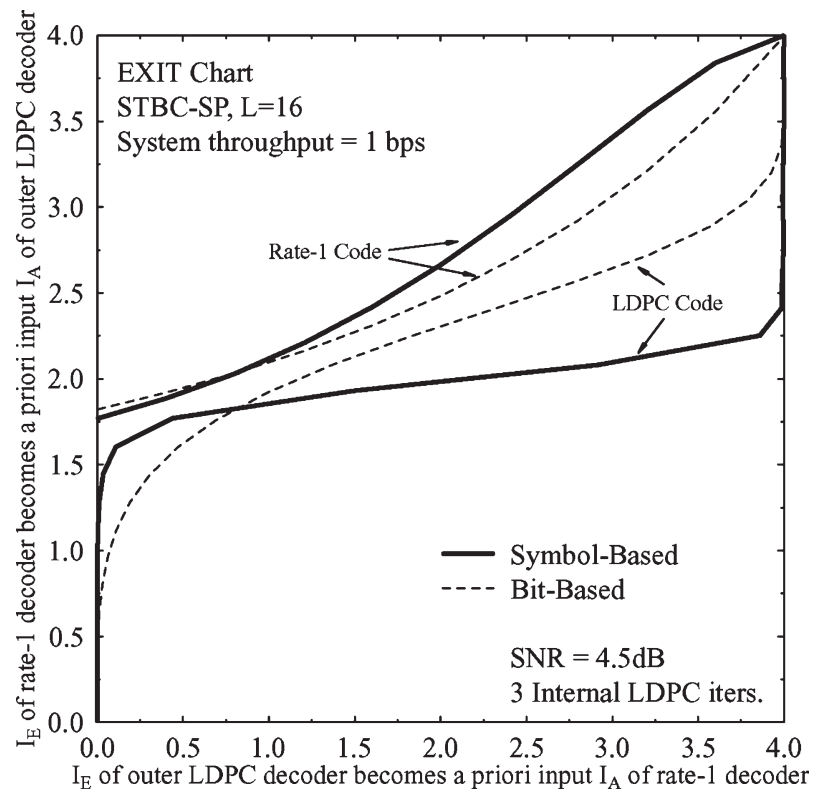

Fig. 3. EXIT chart of symbol-based and bit-based LDPC-coded STBC-SP schemes in combination with the 1/2-rate outer LDPC code defined over $G F(16)$ and $G F(2)$, respectively, using three internal LDPC iterations and the system parameters outlined in Table I.

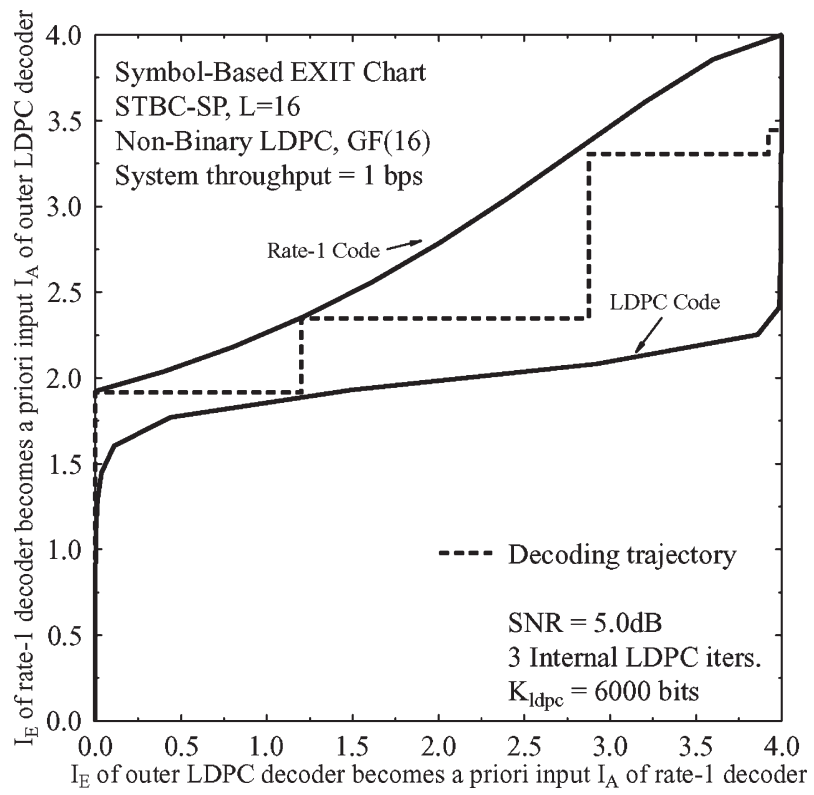

Fig. 4. Decoding trajectory of the symbol-based 1/2-rate nonbinary LDPCcoded [7] STBC-SP scheme defined over $G F(16)$ in combination with the system parameters outlined in Table I and operating at $E_{b} / N_{0}=5.0 \mathrm{~dB}$ after five joint external iterations and three internal LDPC iterations.

and hence, the required $L$ SP symbols were chosen according to the minimum energy and the highest MED criterion proposed in [14].

Fig. 4 illustrates the actual decoding trajectory of the turbo-detected, symbol-based nonbinary LDPC-coded STBC-SP scheme in Fig. 3 when operating at $\mathrm{SNR}=5.0 \mathrm{~dB}$ after $I_{\text {ext }}=5$ joint external iterations and $I_{\text {int }}=3$ internal LDPC iterations. The "zigzag path" shown in Fig. 4 represents the actual EXIT between the rate-1 inner decoder and the outer nonbinary LDPC decoder at SNR $=5.0 \mathrm{~dB}$. The deviation of the decoding trajectory from the prediction of the EXIT chart is due to the fact that a finite LDPC output block length of $K_{\mathrm{ldpc}}=6000$ bits is employed, rendering the assumption of having

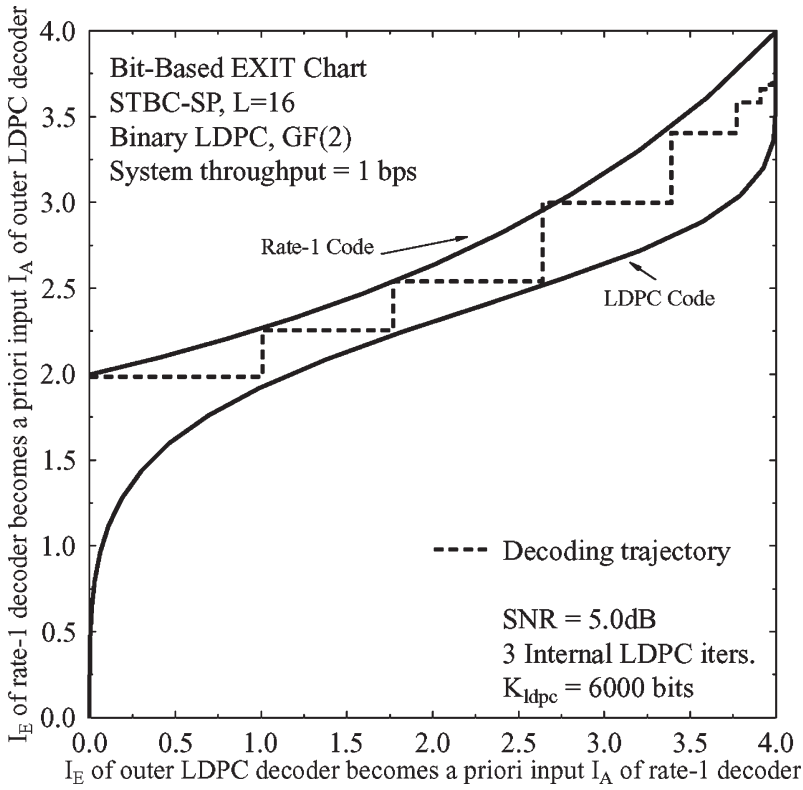

Fig. 5. Decoding trajectory of the bit-based 1/2-rate binary LDPC-coded [5] STBC-SP scheme in combination with the system parameters outlined in Table I and operating at $E_{b} / N_{0}=5.0 \mathrm{~dB}$ after 15 joint external iterations and three internal LDPC iterations.

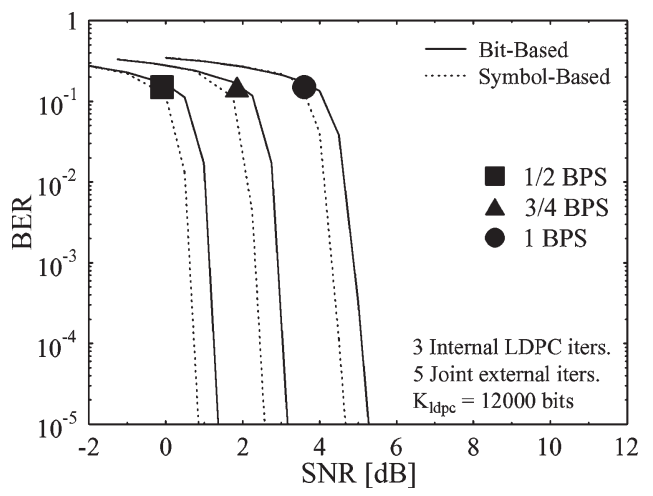

Fig. 6. Performance of symbol-based and bit-based LDPC-coded STBC-SP schemes in combination with the system parameters outlined in Table I after five joint external iterations and three internal LDPC iterations when using an LDPC output block length of $K_{\text {ldpc }}=12000$.

Gaussian-distributed symbol probabilities only approximately valid. This assumption was exploited when creating $\mathbf{A}_{\mathrm{urc}}$ and $\mathbf{A}_{\mathrm{ldpc}}$ for the sake of generating the appropriate a priori information $I_{A}$ to characterize the EXIT characteristics of the constituent decoders. Fig. 5 illustrates the decoding trajectory of the turbo-detected, bitbased binary LDPC-coded STBC-SP scheme of Fig. 3 when operating at $\mathrm{SNR}=5.0 \mathrm{~dB}$ after $I_{\text {ext }}=15$ joint external iterations and $I_{\text {int }}=3$ internal LDPC iterations. Observe in Figs. 4 and 5 that more joint external iterations are required by the bit-based scheme to converge than by the symbol-based scheme.

Fig. 6 compares the attainable performance of both the symbolbased nonbinary LDPC-based [7] and the bit-based binary LDPCcoded [5] STBC-SP schemes using the system parameters in Table I after $I_{\text {ext }}=5$ joint external iterations and $I_{\text {int }}=3$ internal LDPC iterations when using an LDPC output block length of $K_{\text {ldpc }}=$ 12000 bits. Observe in Fig. 6 that the symbol-based LDPC-coded STBC-SP schemes require lower SNR values to achieve a BER of $10^{-5}$, as compared with the bit-based schemes, when using the system 
parameters in Table I and using three internal LDPC iterations as well as an LDPC output block length of $K_{\text {ldpc }}=12000$ bits.

\section{CONCLUSION}

In this paper, we have proposed a novel symbol-based iterative scheme that exploits the advantages of nonbinary LDPC codes [7], the rate-1 inner codes in [8], and the STBC-SP scheme in [4]. Our investigations have demonstrated that attractive performance improvements may be achieved by the proposed scheme over the equivalentthroughput bit-based schemes. Subsequently, novel nonbinary EXIT charts were used to study the convergence of the proposed symbolbased scheme. By contrast, binary EXIT charts were used to explore the convergence of the bit-based binary LDPC-coded STBC-SP schemes. Again, it was demonstrated both by EXIT chart analysis and by the corresponding BER performance curves that the symbol-based scheme is capable of outperforming its bit-based counterpart, and both designs had an edge over Alamouti's now classic STBC scheme, dispensing with the SP-based joint design of the QPSK ST symbols. Our future research will consider similar differentially encoded lowcomplexity SP designs for the sake of requiring no channel estimation as well as ST-equalized systems.

\section{ACKNOWLEDGMENT}

The authors would like to thank the anonymous reviewers for their insightful comments.

\section{REFERENCES}

[1] L. Hanzo, T. H. Liew, and B. L. Yeap, Turbo Coding, Turbo Equalisation and Space-Time Coding: For Transmission Over Fading Channels. Chichester, U.K.: Wiley, 2002.

[2] S. Alamouti, "A simple transmit diversity technique for wireless communications," IEEE J. Sel. Areas Commun., vol. 16, no. 8, pp. 1451-1458, Oct. 1998.

[3] V. Tarokh, H. Jafarkhani, and A. Calderbank, "Space-time block codes from orthogonal designs," IEEE Trans. Inf. Theory, vol. 45, no. 5, pp. 1456-1467, Jul. 1999

[4] W. Su, Z. Safar, and K. J. R. Liu, "Space-time signal design for timecorrelated Rayleigh fading channels," in Proc. IEEE ICC, Anchorage, AK, 2003, vol. 5, pp. 3175-3179.

[5] R. Gallager, "Low-density parity check codes," IEEE Trans. Inf. Theory, vol. IT-8, no. 1, pp. 21-28, Jan. 1962.

[6] C. Berrou, A. Glavieux, and P. Thitimajshima, "Near Shannon limit error-correcting coding and decoding: Turbo-codes," in Proc. IEEE ICC, Geneva, Switzerland, May 1993, pp. 1064-1070.

[7] M. C. Davey and D. J. C. MacKay, "Low density parity check codes over GF (q)," IEEE Commun. Lett., vol. 2, no. 6, pp. 165-167, Jun. 1998.

[8] D. Divsalar, S. Dolinar, and F. Pollara, "Serial concatenated trellis coded modulation with rate-1 inner code," in Proc. IEEE GLOBECOM, San Francisco, CA, Nov. 27-Dec. 1, 2000, vol. 2, pp. 777-782.

[9] B. Scanavino, G. Montorsi, and S. Benedetto, "Convergence properties of iterative decoders working at bit and symbol level," in Proc. IEEE GLOBECOM, San Antonio, TX, 2001, vol. 2, pp. 1037-1041.

[10] A. Grant, "Convergence of non-binary iterative decoding," in Proc. IEEE GLOBECOM, San Antonio, TX, Nov. 2001, vol. 2, pp. 1058-1062.

[11] J. Kliewer, S.-X. Ng, and L. Hanzo, "Efficient computation of EXIT functions for nonbinary iterative decoding," IEEE Trans. Commun., vol. 54, no. 12 , pp. 2133-2136, Dec. 2006.

[12] T. J. Richardson and R. Urbanke, "The capacity of low-density paritycheck codes under message-passing decoding," IEEE Trans. Inf. Theory, vol. 47, no. 2, pp. 599-618, Feb. 2001.

[13] J. H. Conway and N. J. Sloane, Sphere Packings, Lattices and Groups. New York: Springer-Verlag, 1999.

[14] O. Alamri, B. L. Yeap, and L. Hanzo, "A turbo detection and spherepacking-modulation-aided space-time coding scheme," IEEE Trans. Veh. Technol., vol. 56, no. 2, pp. 575-582, Mar. 2007.
[15] L. Bahl, J. Cocke, F. Jelinek, and J. Raviv, "Optimal decoding of linear codes for minimizing symbol error rate," IEEE Trans. Inf. Theory, vol. IT-20, no. 2, pp. 284-287, Mar. 1974.

[16] S. ten Brink, "Designing iterative decoding schemes with the extrinsic information transfer chart," AEÜ Int. J. Electron. Commun., vol. 54, no. 6, pp. 389-398, Nov. 2000.

[17] I. Land, P. Hoeher, and S. Gligorevic, "Computation of symbol-wise mutual information in transmission systems with $\log$ APP decoders and application to EXIT charts," in Proc. Int. ITG Conf. SCC, Erlangen, Germany, Jan. 2004, pp. 195-202.

\section{Recovering Signal Energy From the Cyclic Prefix in OFDM}

Gregory E. Bottomley, Fellow, IEEE, and

Leif R. Wilhelmsson, Senior Member, IEEE

\begin{abstract}
In orthogonal frequency-division multiplexing (OFDM) systems, a cyclic prefix (CP) is often added at the transmitter and discarded at the receiver. When the length of the $\mathrm{CP}$ exceeds the delay spread of the channel, a portion of the CP can be used to recover additional signal energy. In the past, Nyquist windowing techniques have been proposed to recover signal energy, thereby improving performance. In this paper, linear maximum-likelihood (ML) and minimum-mean-square-error (MMSE) approaches are developed, which further improve performance, particularly when the signal-to-noise ratio (SNR) is low. Simpler twovalued windowing (TVW) solutions are also provided, which generally employ one or two non-Nyquist windows.
\end{abstract}

Index Terms-Cyclic prefix (CP), demodulation, maximum-likelihood (ML) detection, multicarrier transmission, orthogonal frequency-division multiplexing (OFDM).

\section{INTRODUCTION}

Orthogonal frequency-division multiplexing (OFDM), which was originally developed in the late 1950s and 1960s (see references in [1]), is being used or considered in a variety of wireless communication systems. With OFDM, a block of symbols is sent in parallel on different frequency subcarriers. Wireless communication channels are often dispersive, introducing interference between and within blocks of symbols. Ideally, all symbols should be jointly detected [2], which requires significant receiver complexity. To simplify receiver processing, part of the block of symbols is copied and preappended at the transmitter, forming a "cyclic prefix" (CP) [3]. At the receiver, this portion of the signal is typically discarded, thus avoiding interblock interference and allowing for simple subcarrier separation using a fast Fourier transform (FFT).

The CP may be longer than is needed for a particular user's receiver. For broadcast, the $\mathrm{CP}$ should be designed for the worst-case delay spread, which typically is not experienced by all users. For multipleaccess, where different subcarriers are allocated to different users, the $\mathrm{CP}$ should be designed for the user with the largest delay spread. In

Manuscript received January 30, 2007; revised August 22, 2007 and October 29, 2007. This work was presented in part at the IEEE Vehicular Technology Conference, Montréal, QC, Canada, September 25-28, 2006. The review of this paper was coordinated by Prof. H. Nguyen.

G. E. Bottomley is with Ericsson Inc., Research Triangle Park, NC 27709 USA.

L. R. Wilhelmsson is with Ericsson AB, Lund, Sweden.

Color versions of one or more of the figures in this paper are available online at http://ieeexplore.ieee.org.

Digital Object Identifier 10.1109/TVT.2007.914057 